\title{
On the Convolution of Functions of $\Lambda_{p}-$ Bounded Variation and a Locally Compact Hausdorff Topological Group
}

\author{
S. M. Nengem ${ }^{1}$, D. Samaila ${ }^{1}$ and M. Solomon ${ }^{1}$ \\ ${ }^{1}$ Department of Mathematical Sciences, Adamawa State University, P. M. B. 25, Mubi, Nigeria
}

\begin{abstract}
The smoothness-increasing operator "convolution" is well known for inheriting the best properties of each parent function. It is also well known that if $f \in L^{l}$ and $g$ is a Bounded Variation (BV) function, then $f$ * $g$ inherits the properties from the parent's spaces. This aspect of $B V$ can be generalized in many ways and many generalizations are obtained. However, in this paper we introduce the notion of $\Lambda_{p}-$ Bounded variation function. In relation to that we show that the convolution of two functions $f * g$ is the inverse Fourier transforms of the two functions. Moreover, we prove that if $f, g \in \Lambda B V^{(p)}[0,2 \pi]$, then $f * g \in \Lambda B V^{(p)}[0,2 \pi]$, and that on any locally compact Abelian group, a version of the convolution theorem holds.
\end{abstract}

Keywords: Convolution, $\Lambda_{p}$-Bounded variation, Fourier transform, Abelian group.

\section{Introduction}

In mathematics and, in particular, functional analysis, convolution is a mathematical operation on two functions $f$ and $g$, producing a third function that is typically viewed as a modified version of one of the original functions, giving the area overlap between the two functions as a function of the amount that one of the original functions is translated. Convolution is similar to cross-correlation. It has applications that include probability, statistics, computer vision, image and signal processing [9], electrical engineering and differential equations. The convolution can be defined for functions on groups other than Euclidean space. For example, periodic functions, such as the discrete-time Fourier transform, can be defined on a circle and convolved by periodic convolution. And discrete convolution can be defined for functions on the set of integers. Generalizations of convolution have applications in the field of numerical analysis and numerical linear algebra, and in the design and implementation of finite impulse response filters in signal processing. Computing the inverse of the convolution operation is known as deconvolution.

According to origin and history of convolution [10], "Probably one of the first occurrences of the real convolution integral took place in the year 1754 when the mathematician Jean-le-Rond D'Alembert derived Taylor's expansion theorem on page 50 of Volume 1 of his book "Recherches sur differents points importants du systeme du monde".

Also, an expression of the type:

$$
\int f(u) \cdot g(x-u) d u
$$

is used by Sylvestre Francois Lacroix on page 505 of his book entitled Treatise on differences and series, which is the last of 3 volumes of the encyclopedic series: "Traité du calcul différentiel et du calcul intégral", Chez Courcier, Paris, 1797-1800 [14]. Soon thereafter, convolution operations appear in the works of Pierre Simon Laplace, Jean Baptiste Joseph Fourier, Simeon Denis Poisson, and others. The term itself did not come into wide use until the 1950s or 60s. Prior to that, it was sometimes known as faltung (which means folding in German), composition product, superposition integral, and Carson's integral. Yet it appears as early as 1903, though the definition is rather unfamiliar in older uses [1], [2].

The operation:

$$
\int_{0}^{t} \varphi(s) \psi(t-s) d s, \quad 0 \leq t<\infty,
$$

is a particular case of composition products considered by the Italian mathematician Vito Volterra in 1913 [3]. In the investigation of convergence of Fourier series, Waterman introduced the class of functions of $\Lambda \mathrm{BV}$ in the early seventies. In 1980 Shiba [8] generalized this class. He introduced the class $\Lambda B V^{(p)}$. In 2006, Vyas [12] show that the functions of generalized bounded variation inherit some properties of bounded variation under convolution.

Our aim in this paper is to study and analyze the convolution algebra and then consider the functions of $\Lambda_{\mathrm{p}}$ - bounded variation under the same umbrella. This will guide us in our next article, to understand why the convolution produces the output of an LTI (Linear time-invariant) system, and why the behavior of a linear, continuous-time, time-invariant system with input signal $\mathrm{x}(\mathrm{t})$ and output signal $\mathrm{y}(\mathrm{t})$ is described by the 
convolution integral [6], leading to the study of Linear time-invariant theory, commonly known as LTI system theory.

\section{Preliminary}

Definition 1. Given an interval $I$, a sequence of non-decreasing positive real numbers $\Lambda=\left\{\lambda_{n}\right\},(n=1,2, \ldots)$ such that $\sum_{n=1}^{\infty} \frac{1}{\lambda_{n}}$ diverges and $1 \leq p<\infty$. We say that $f \in \Lambda B V^{(\mathrm{p})}(I)$

(that is $f$ is of $p-\Lambda-$ bounded variation on $I$ ) if

$$
V_{\Lambda}(f, p, I)=\sup _{\left\{I_{n}\right\}} V_{\Lambda}\left(\left\{I_{n}\right\}, f, p, I\right)<\infty,
$$

Where $V_{\Lambda}\left(\left\{I_{n}\right\}, f, p, I\right)=\left(\sum_{n} \frac{\left|f\left(a_{n}\right)-f\left(b_{n}\right)\right|^{p}}{\lambda_{n}}\right)^{1 / p}$, and $\left\{I_{n}\right\}$ is a sequence of non-overlapping subintervals $I_{n}=$ $\left[a_{n}, b_{n}\right] \subset I=[0,2 \pi]$. With this metric as a function of the set, we defines a functional $V_{\wedge_{p}}(\cdot)$, given by

$$
V_{\Lambda_{p}}(f)^{1 / p}=\sup \left(\sum_{n=1}^{\infty} \frac{\left|f\left(I_{n}\right)\right|^{p}}{\lambda_{n}}\right)^{1 / p}<\infty, \forall f \in \Lambda B V^{(\mathrm{p})} \cdot[7] .
$$

Note that if $p=1$ one get the class $\Lambda B V(I)$; if $\lambda_{n}=1$ for all $n$, one gets the class $B V^{(\mathrm{p})}$; if $p=1$ and $\lambda_{n}=$ $n$, for all $n$, one gets the class $H B V(I)$; if $p=1$ and $\lambda_{n}=1$ for all $n$, one gets the class $B V(I)$.

Definition 2. For $p \geq 0,0<\alpha \leq 1$, we say that $f \in \operatorname{Lip}(\alpha, p)$ over I if

$$
\left\|T_{y} f-f\right\|_{p, I}=0\left(|h|^{\alpha}\right) \text { as } h \rightarrow 0
$$

Where $\|(\cdot)\|_{p, \mathrm{I}}$ denotes the $L^{p}$ norm over $I$ and $T_{h} f(x)=f(x+h)$, [11]. It makes sense to consider the integral modulus of continuity

$$
\omega_{p}(\delta, f):=\sup _{0 \leq h \leq \delta}\left(\int_{0}^{1-h}|f(t+h)-f(t)|^{p}\right)^{1 / p} d t
$$

For $0 \leq \delta \leq 1$. However, if $f$ is define on $R$ instead of $[0,2 \pi]$ and if $f$ is $2 \pi-$ periodic, it is convenient to modify the definition and put

$$
\omega_{p}(\delta, f):=\sup _{0 \leq h \leq \delta}\left(\int_{0}^{2 \pi}|f(t+h)-f(t)|^{p}\right)^{1 / p} d t
$$

Definition 3. Let $f$ be a $2 \pi$-periodic function, $f$ is of $\Lambda$ - bounded variation on $[0,2 \pi]$, then the Fourier transform of $f$ is given by

$$
\mathrm{F}[f(x)]=\int_{0}^{2 \pi} f(t) e^{-i t x} d x=f(t), t \in[0,2 \pi] .[4]
$$

In Fourier analysis, for any $2 \pi$ - periodic function $f$ and $g$, $f$ convolution $g$ is defined as follows:

Definition 4. For any $f, g \in L^{1}[0,2 \pi], f * g$ is define as

$$
(f * g)(x)=\frac{1}{2 \pi} \int_{0}^{2 \pi}(f(x-y) g(y) d y . \text { [12] }
$$

In view of the above definition, we state the convolution theorem as in theorem 1 below. The approach adapted in the formulation and proof of the theorem is in accordance with Dass, (2006) [5] Laplace transformation.

Definition 5. For a positive integer $\mathrm{r}$, we say that $f \in r-B V(I)$ ( that is, $f$ is of bounded $r^{t h}$ - variation over $I$ ) if for arbitrary $(n+1)$ - points $x_{1}<x_{2}<\cdots<x_{n}$ in $I$, in an arithmetic progression we have $V^{r}(f, I)=\sup _{n} V_{n}^{r}(f, I)<\infty$, where $\sup _{n} V_{n}^{r}(f, I)=\sum_{i=0}^{n-r}\left|\Delta^{r} f\left(x_{i}\right)\right|,[12]$

In which $\Delta f\left(x_{i}\right)=f\left(x_{i+1}\right)-f\left(x_{i}\right)$ and for some $k \geq 2$

$$
\begin{aligned}
& \Delta^{k} f\left(x_{i}\right)=\Delta^{k-1} f\left(x_{i+1}\right)-\Delta^{k-1} f\left(x_{i}\right), \text { so that } \\
& \Delta^{r} f\left(x_{i}\right)=\sum_{m=0}^{r}(-1)^{m}\left(\begin{array}{c}
r \\
m
\end{array}\right) f\left(x_{i+r-m}\right)
\end{aligned}
$$

\section{Algebraic Properties}

The convolution defines a product on the linear space of integrable functions. This product satisfies the following algebraic properties, which formally mean that the space of integrable functions with the product given by convolution is a commutative algebra without identity [13]. Other linear spaces of functions, such as the space of continuous functions of compact support, are closed under the convolution, and so also form commutative algebras.

Commutativity: $f * g=g * f$

Associativity: $f *(g * h)=(f * g) * h$

Distributive: $f *(g+h)=(f * g)+(f * h)$

Associativity with scalar multiplication: $a(f * g)=(a f) * g$ for any real (or complex) number $a$.

No algebra of functions possesses an identity for the convolution. The lack of identity is typically not a major inconvenience since most collections of functions on which the convolution is performed can be convolved with a delta distribution, or, at the very least (as is the case of $L^{1}$ ) admit approximations to the identity. The linear space of compactly supported distributions does, however, admit an identity under the convolution. Specifically,

$$
f * \delta=f
$$


where $\delta$ is the delta distribution.

Some distributions have an inverse element for the convolution, $\mathrm{S}^{(-1)}$, which is defined by $\mathrm{S}^{(-1)} * \mathrm{~S}=\delta$

The set of invertible distributions forms an Abelian group under the convolution.

\section{Functions Of $\Lambda_{\mathbf{P}}-$ Bounded Variation}

Lemma 1. If $f \in L^{1}[0,2 \pi]$ and $g \in L^{p}[0,2 \pi](p \geq 1)$, then,

$\left\|T_{h} f * g-f * g\right\|_{p,[0,2 \pi]} \leq\|f\|_{1}\left\|T_{h} g-g\right\|_{p,[0,2 \pi]} .[11]$

Proof: For any $h \in L^{p}[0,2 \pi]$, where $q$ satisfies $\frac{1}{p}+\frac{1}{q}=1$, from the Fubini - Tonelli theorem we get

$$
\begin{aligned}
& \left|\frac{1}{2 \pi} \int_{0}^{2 \pi}\left[T_{h} f * g(x)-f * g(x)\right] h(x) d x\right| \\
& \leq \frac{1}{2 \pi} \int_{0}^{2 \pi}|h(x)|\left\{\frac{1}{2 \pi} \int_{0}^{2 \pi}\left|f(y) \|\left(T_{h} g-g\right)(x-y)\right| d y\right\} d x \\
& =\frac{1}{2 \pi} \int_{0}^{2 \pi}|f(y)|\left\{\frac{1}{2 \pi} \int_{0}^{2 \pi}\left|h(x) \|\left(T_{h} g-g\right)(x-y)\right| d x\right\} d y \\
& =\|f\|_{1}\|h\|_{q}\left\|T_{h} g-g\right\|_{p}, \text { (by Holder's inequality). }
\end{aligned}
$$

Hence the result follows.

Theorem 1. If the inverse Fourier transforms of two functions $f(x)$ and $g(x)$ is $F(t)$ and $G(t)$ respectively (that is $F(t)=F^{-1}[f(x)]$, and $G(t)=F^{-1}[g(x)]$ ), then

$$
F^{-1}[f(x) g(x)]=\frac{1}{2 \pi} \int_{0}^{t} G(t-u) F(u) d u=F * G, 0 \leq t \leq 2 \pi .
$$

Where $F * G$ is known as convolution or Faltung of $\mathrm{F}$ and $\mathrm{G}$ [5].

Proof: Our proof here uses Dass as a basis. From the conditions of the theorem we have; $F[F(t)]=$ $f(x)$ and $F[G(t)]=g(x)$, therefore

$$
\begin{aligned}
& f(x) g(x)=F[f(t)] \cdot F[g(t)]=\frac{1}{2 \pi} \int_{0}^{2 \pi} F(z) e^{-i x z} d z \frac{1}{2 \pi} \int_{0}^{2 \pi} G(y) e^{-i x y} d y \\
& =\iint_{0}^{2 \pi} e^{-i x(z+y)} F(z) G(y) d z d y \\
& =\iint_{[0,2 \pi]} e^{-i x(z+y)} F(z) G(y) d z d y
\end{aligned}
$$

Now we take the positive quadrant on the plane over which the double integration extends. However to transform the double integration from the region $R(x, y)$ to another, we consider $z=w, y=v-w$
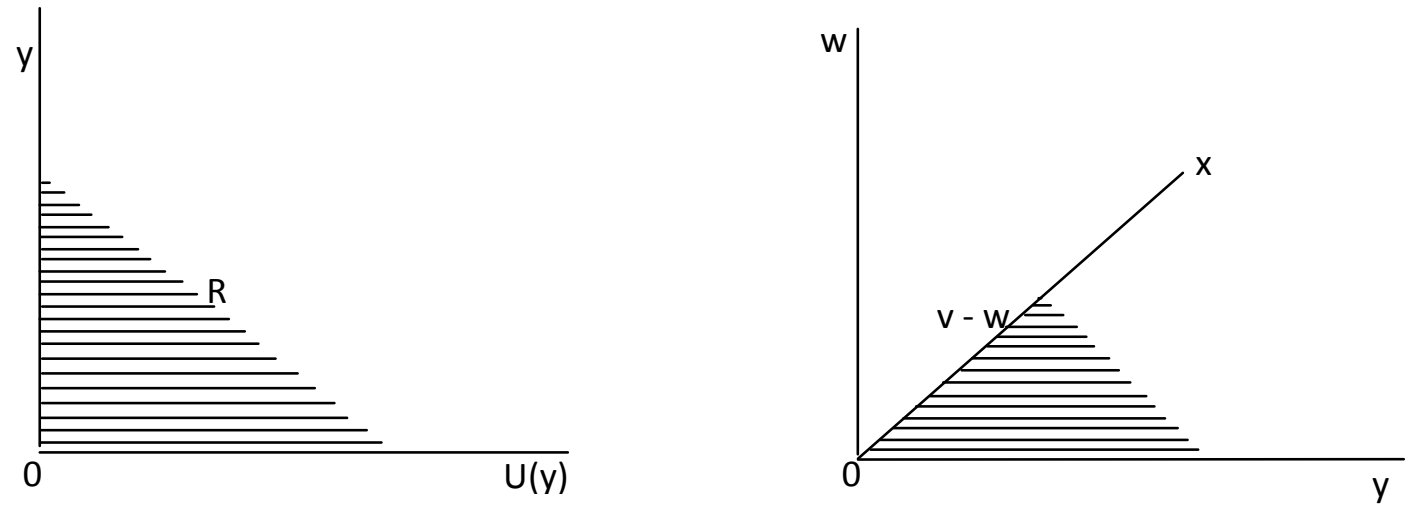

when $z=0, w=0$, when $z=\infty, w=0$ and when $y=0, v-w=0, y=\infty,(v-w)=\infty$. The Jacobian of the transformation is

$$
\frac{\partial(z, y)}{\partial(v, w)}=\left|\begin{array}{ll}
\frac{\partial z}{\partial v} & \frac{\partial y}{\partial v} \\
\frac{\partial z}{\partial w} & \frac{\partial y}{\partial w}
\end{array}\right|=\left|\begin{array}{cc}
0 & 1 \\
1 & -1
\end{array}\right|=-1 .
$$

Also given $d z d y=d v d w$. Thus equation (1) becomes

$$
\iint_{[0,2 \pi]} e^{-i x v} F(w) G(v-w) d v d w=\int_{0}^{2 \pi} e^{-i x v}\left[\int_{0}^{v} F(w) G(v-w) d w\right] d v
$$

On replacing the variables $v$ by $t$ and $w$ by $y$ we have

Hence

$$
\int_{0}^{2 \pi} e^{-i x t}\left[\int_{0}^{t} F(y) G(t-y) d y\right] d x=F\left\{\int_{0}^{t} F(y) G(x-y) d y\right\}=F[F * G]
$$

$$
f(x) g(x)=F[f(t)] \cdot F[g(t)]=\frac{1}{2 \pi} \int_{0}^{2 \pi} F(t-u) G(u) d u=F[F * G] .
$$

Remark: By lemma 1, for every sequence $\left\{I_{k}\right\}_{k=1}^{2 n}$ of non overlapping sub-intervals $I_{k}=\left[a_{k} b_{k}\right] \subset I=[0,2 \pi]$,

$$
F[F * G]=\frac{1}{2 \pi} \int_{0}^{2 \pi}|g(u)|\left(\sum_{k=1}^{2 n} \frac{\left|f\left(b_{k}-u\right)-f\left(a_{k}-u\right)\right|}{\lambda_{k}}\right) d u, 0 \leq t \leq 2 \pi
$$




$$
=V_{\Lambda}(f,[0,2 \pi])\|g\| \text {. Hence } F[F * G] \in \Lambda B V .
$$

Theorem 2: If $f$ and $g$ are two functions in $\Lambda B V^{(p)}[0,2 \pi](p \geq 1)$, then $f * g \in \Lambda B V^{(p)}[0,2 \pi]$, [12].

Proof: Since every function of $\Lambda B V^{(p)}$ is bounded, from lemma 1, we get

$$
\int_{0}^{2 \pi}\left|(f * g)\left(I_{k}\right)\right|^{p} d x \leq\|f\|^{p} \int_{0}^{2 \pi}\left|g\left(I_{k}\right)\right|^{p} d x
$$

for every sequence of non overlapping subintervals $I_{k}=\left[a_{k}, b_{k}\right] \subset I=[0,2 \pi]$. Dividing both sides of the above equation by $\lambda_{k}$ and performing summation from $k=1$ to $\infty$, we get

$$
\begin{aligned}
& \int_{0}^{2 \pi}\left(\sum_{1}^{\infty} \frac{\left|(f * g)\left(I_{k}\right)\right|^{p}}{\lambda_{k}}\right) d x \leq\|f\|_{\infty}^{p} \int_{0}^{2 \pi}\left(\sum_{k=1}^{\infty} \frac{\left|g\left(I_{k}\right)\right|^{p}}{\lambda_{k}}\right) d x \\
& =2 \pi V_{\Lambda}\left(g, p,\left\{I_{k}\right\}\right)\|f\|_{\infty}^{p} .
\end{aligned}
$$

Hence the result follows.

Theorem 3: If $f \in L^{1}[0,2 \pi]$ and $g \in r-B V[0,2 \pi]$, then $f * g \in r-B V[0,2 \pi]$, [12].

Proof: From the definition of convolution we get

$$
\begin{aligned}
& \Delta(f * g)(x)=\frac{1}{2 \pi} \int_{0}^{2 \pi} f(y) \Delta g(x-y) d y, \\
& \Delta^{2}(f * g)(x)=\frac{1}{2 \pi} \int_{0}^{2 \pi} f(y) \Delta^{2} g(x-y) d y,
\end{aligned}
$$

Similarly, for any positive integer $r$, we have

$$
\Delta^{r}(f * g)(x)=\frac{1}{2 \pi} \int_{0}^{2 \pi} f(y) \Delta^{r} g(x-y) d y,
$$

Thus for arbitrary $(n+1)$ - points; $x_{0}<x_{1}<x_{2}<\cdots<x_{n}$ in $I=[0,2 \pi]$, in an arithmetic progression we get $\left|\Delta^{r}(f * g)\left(x_{k}\right)\right| \leq \frac{1}{2 \pi} \int_{0}^{2 \pi}\left|f(y) \| \Delta^{r} g\left(x_{k}-y\right)\right| d y, \forall k=0, n-r$.

By taking $k=0,1,2, \ldots, n-r$, we get

$$
\begin{gathered}
\sum_{k=0}^{n-r}\left|\Delta^{r}(f * g)\left(x_{k}\right)\right| \leq \frac{1}{2 \pi} \int_{0}^{2 \pi}|f(y)|\left(\sum_{k=0}^{n-r}\left|\Delta^{r} g\left(x_{k}-y\right)\right|\right) d y, \\
\leq V^{r}(g,[0,2 \pi])\|f\|_{1}
\end{gathered}
$$

Remark 1. By theorem 1, we see that the operation $f * g$ is commutative thus

$$
f * g=\frac{1}{2 \pi} \int_{0}^{2 \pi}(f(t-u) g(u) d u,
$$

when $t-u=z$ we get

$$
f * g=\frac{1}{2 \pi} \int_{0}^{2 \pi}\left(f(z) g(t-z) d z=\frac{1}{2 \pi} \int_{0}^{2 \pi}(g(t-z) f(z) d z=g * f\right.
$$

Moreover this theorem may best be restated as "if $f(x)$ and $g(x)$ are the Fourier transforms of two functions $F(t)$ and $G(t)$ that are sectionally continuous on each interval $0 \leq t \leq u$, then the transform of the convolution $F(t) * G(t)$ exist and it is $f(x) \cdot g(x)$ ".

Remark 2. Dividing both sides of equation 3 by $\lambda_{k}$ we get

$$
\begin{aligned}
\sum_{k=0}^{n-r} \frac{\left|\Delta^{r}(f * g)\left(x_{k}\right)\right|}{\lambda_{k}} & \leq \frac{1}{2 \pi} \int_{0}^{2 \pi}|f(y)|\left(\sum_{k=0}^{n-r} \frac{\left|\Delta^{r} g\left(x_{k}-y\right)\right|}{\lambda_{k}}\right) d y, \\
& \leq V_{\Lambda}^{r}\left(g,[0,2 \pi]\|f\|_{1}\right.
\end{aligned}
$$

Hence we say that $f * g$ is also of $\Lambda-$ bounded variation.

\section{Convolutions On Groups}

If $G$ is a suitable group endowed with a measure $\lambda$, and if $f$ and $g$ are real or complex valued integrable functions on $G$, then we can define their convolution by

$$
(f * g)(x)=\int_{G} f(y) g\left(y^{-1} x\right) d \lambda(y) .
$$

In typical cases of interest $G$ is a locally compact Hausdorff topological group and $\lambda$ is a (left-) Haar measure. In that case, unless $G$ is unimodular, the convolution defined in this way is not the same as

$$
\int f\left(x y^{-1}\right) g(y) d \lambda(y) \text {. }
$$

The preference of one over the other is made so that convolution with a fixed function $g$ commutes with left translation in the group:

$$
L_{h}(f * g)=\left(L_{h} f\right) * g=f *\left(L_{h} g\right) .
$$

Furthermore, the convention is also required for consistency with the definition of the convolution of measures given below. However, with a right instead of a left Haar measure, the latter integral is preferred over the former.

On locally compact Abelian groups, a version of the convolution theorem holds: the Fourier transform of a convolution is the point-wise product of the Fourier transforms. The circle group $\mathbf{T}$ with the Lebesgue measure is an immediate example. For a fixed $g$ in $L^{1}(\mathbf{T})$, we have the following familiar operator acting on the Hilbert space $L^{2}(\mathbf{T})$ :

$$
T f(x)=\frac{1}{2 \pi} \int_{T} f(y) g(x-y) d y
$$

The operator $T$ is compact. A direct calculation shows that its adjoint $T^{*}$ is convolution with $\bar{g}(-y)$. 
By the commutativity property cited above, $T$ is normal, i.e. $T^{*} T=T T^{*}$. Also, $T$ commutes with the translation operators. Consider the family $S$ of operators consisting of all such convolutions and the translation operators. Then $S$ is a commuting family of normal operators. According to spectral theory, there exists an orthonormal basis $\left\{h_{k}\right\}$ that simultaneously diagonalizes S. This characterizes convolutions on the circle. Specifically, we have

$$
h_{k}(x)=e^{i k x}, \quad k \in Z,
$$

which are precisely the characters of $\mathbf{T}$. Each convolution is a compact multiplication operator in this basis. This can be viewed as a version of the convolution theorem discussed above.

A discrete example is a finite cyclic group of order $n$. Convolution operators are here represented by circulant matrices, and can be diagonalized by the discrete Fourier transform.

A similar result holds for compact groups (not necessarily abelian): the matrix coefficients of finite-dimensional unitary representations form an orthonormal basis in $L^{2}$ by the Peter-Weyl theorem, and an analog of the convolution theorem continues to hold, along with many other aspects of harmonic analysis that depend on the Fourier transform.

\section{Conclusion}

In this paper we examine the notion of functions of $\Lambda_{\mathrm{p}}$-bounded variation under well known convolution operator. Some selected properties of functions of $\Lambda_{\mathrm{p}}$-bounded variation are shown to inherit the properties of the parents function. Perhaps the most important and interesting property is the fact that if $f$ and $g$ are two functions of $\Lambda_{\mathrm{p}}$-bounded variation (i.e. $f, g \in \Lambda \mathrm{BV}^{(\mathrm{p})}[0,2 \pi]$ ), then $f * g$ also belong to the same space. Another interesting issue is that the functions of $\Lambda_{\mathrm{p}}$-bounded variation are shown to be commutative under the convolution operator. The convolution theory is best stated in a simpler and understanding language for interesting readers. i.e. If $f(x)$ and $g(x)$ are the Fourier transforms of two functions $\mathrm{F}(t)$ and $\mathrm{G}(t)$ and are sectionally continuous on each interval $0 \leq t \leq u$, then the transform of the convolution $\mathrm{F}(t) * \mathrm{G}(t)$ exist and is $f(x) \cdot g(x)$. Furthermore, on any locally compact Abelian group, a version of the convolution theorem holds.

Note that we have not completely extracts the important facts about the properties of the convolution operator. Any of the properties can be explored in more depth by any interesting reader, most especially; there are other properties which are not considered in this paper due to time space.

\section{References}

[1] Dominguez-Torres, Alejandro, "Origin and history of convolution". 2010. http://www.slideshare.net/Alexdfar/origin-adn-history-ofconvolution. Cranfield, Bedford MK43 OAL, UK. Retrieved Mar 13, 2013.

[2] E. Hewitt and A. Ross, Kenneth, Abstract harmonic analysis. Vol. II: Structure and analysis for compact groups. Analysis on locally compact Abelian groups, Die Grundlehren der mathematischen Wissenschaften, Band 152, Berlin, New York, 1970.

[3] E. Hewitt, and A. Ross, Kenneth, Abstract harmonic analysis. Vol. I, Grundlehren der Mathematischen Wissenschaften [Fundamental Principles of Mathematical Sciences] 115 (2nd ed.), Berlin, New York, 1979.

[4] E. Lifeyand and M. Standmuller, On a Hardy-Littlewood Theorem, Department of mathematics Bar-Ilan University, 2013.

[5] H. K. Dass, Advance Engineering Mathematics (Fifteenth Revised Edition), S. Chand \& Company Ltd, 2006.

[6] J. P. Hespanha, Linear System Theory, Princeton university press, 2009.

[7] M. Hormozi, A. A. Ledari \& F. Prus-Wisniowki, On p- $\Lambda$-Bounded Variation, Bulletin of the Iranian Mathematical Society, Vol. 37 No. 4, 2011, pp. 35-49.

[8] M. Shiba, On absolute Convergence of Fourier series of Functions of Class $\Lambda \mathrm{BV}{ }^{(\mathrm{p})}$, Sci. Rep. Fukushima Uni. 30; 1980, 7-10.

[9] P. J. Diggle, A kernel method for smoothing point process data, Journal of the Royal Statistical Society, Series C 34: 138-147

[10] R. Bracewell, The Fourier Transform and Its Applications (2nd ed.), McGraw-Hill, 1986.

[11] R. G. Vyas, On the Convolution of functions of Generalized Bounded Variation, Georgian Mathematical Journal, Volume 13, Number 1, 2006, 193-197, India.

[12] R. G. Vyas, Properties of Functions of Generalized Bounded Variation, Department of mathematics, Faculty of Science, The Maharaja Sarajirao University of Baroda, Vadodara-390002, Gujarat, India, 2006.

[13] R. Strichartz, A Guide to Distribution Theory and Fourier Transforms, CRC Press, 1994.

[14] S. Damelin and W. Miller, The Mathematics of Signal Processing, Cambridge University Press, 2011. 